\title{
Two-Temperature Generalized Thermoviscoelasticity with Fractional Order Strain Subjected to Moving Heat Source: State Space Approach
}

\author{
Renu Yadav, ${ }^{1}$ Kapil Kumar Kalkal, ${ }^{2}$ and Sunita Deswal ${ }^{2}$ \\ ${ }^{1}$ Department of Mathematics, N.M. Govt. College, Hansi, Haryana 125033, India \\ ${ }^{2}$ Department of Mathematics, Guru Jambheshwar University of Science and Technology, Hisar, Haryana 125001, India \\ Correspondence should be addressed to Sunita Deswal; spannu_gju@yahoo.com
}

Received 30 July 2015; Accepted 28 September 2015

Academic Editor: Mario Ohlberger

Copyright (c) 2015 Renu Yadav et al. This is an open access article distributed under the Creative Commons Attribution License, which permits unrestricted use, distribution, and reproduction in any medium, provided the original work is properly cited.

\begin{abstract}
The theory of generalized thermoelasticity with fractional order strain is employed to study the problem of one-dimensional disturbances in a viscoelastic solid in the presence of a moving internal heat source and subjected to a mechanical load. The problem is in the context of Green-Naghdi theory of thermoelasticity with energy dissipation. Laplace transform and state space techniques are used to obtain the general solution for a set of boundary conditions. To tackle the expression of heat source, Fourier transform is also employed. The expressions for different field parameters such as displacement, stress, thermodynamical temperature, and conductive temperature in the physical domain are derived by the application of numerical inversion technique. The effects of fractional order strain, two-temperature parameter, viscosity, and velocity of internal heat source on the field variables are depicted graphically for copper material. Some special cases of interest have also been presented.
\end{abstract}

\section{Introduction}

The classical thermoelasticity theory based on Fourier's law of heat conduction suffers from the deficiency of admitting thermal signals propagating with infinite speed. Numerous alternative theories of heat conduction have been put forth to overcome this deficiency, allowing heat to propagate as wave at finite speed. Among these theories, the extended theory of thermoelasticity proposed by Lord and Shulman [1] involving one relaxation time and the temperature-rate dependent theory of thermoelasticity propounded by Green and Lindsay [2] involving two relaxation times are the earliest and well established theories. Green and Naghdi [3-5] developed a theory where the characteristics of material response for thermal phenomenon are based on three types of constitutive response functions, labelled as types I, II, and III. When the theory of type I is linearized, we obtain the classical system of thermoelasticity. In model II, the internal rate of production of entropy is taken to be identically zero, implying no dissipation of thermal energy. Model III includes the previous two models as special case and admits dissipation of energy in general.

In the late 1960s, Chen and Gurtin [6] and Chen et al. [7, 8] formulated the two-temperature thermoelasticity theory. In this theory, the classical Clausius Duhamel inequality was replaced by another one depending on two temperaturesconductive temperature $\varphi$ and thermodynamical temperature $\theta$. The first is due to the thermal processes and the second is due to the mechanical processes inherent between the particles and layers of elastic materials. Boley and Tolins [9] found that the two temperatures and the strain have representations in the form of a travelling wave pulse response which occurs instantaneously throughout the body. The key element that sets the two-temperature thermoelasticity apart from the classical theory of thermoelasticity is the material parameter $a$, called the temperature discrepancy. Specifically, if $a=0$, then $\varphi=\theta$ and the field equations of classical theory can be fully recovered from the two-temperature thermoelasticity.

Warren and Chen [10] investigated the wave propagation in the two-temperature thermoelasticity. Youssef [11] put 
forward this theory in the context of generalized theory of thermoelasticity. Youssef and Al-Lehaibi [12] studied a onedimensional problem of two-temperature generalized thermoelasticity by employing the state-space technique. They showed that the obtained results are qualitatively different as compared to those in case of one-temperature thermoelasticity. Youssef [13] constructed another two-temperature generalized thermoelasticity theory for a homogeneous and isotropic medium in the context of Green and Naghdi model of type II.

Deswal and Kalkal [14] considered a new model of timefractional derivative in the context of micropolar generalized thermoviscoelasticity theory with two temperatures. Zenkour and Abouelregal [15] employed state-space approach for an infinite medium with a spherical cavity based upon two-temperature generalized thermoelasticity and fractional heat conduction. Othman and Hilal [16] studied a twodimensional problem of thermoelastic rotating material with voids under the effect of gravity and temperature dependent properties employing the two-temperature generalized thermoelasticity in the context of Green-Naghdi theory of types II and III. Bera et al. [17] applied two-temperature generalized thermoelasticity to determine the conductive and thermodynamic temperatures as well as the deformation and stresses in an annular disk.

The fractional calculus has attracted intense attention of an increasing number of mathematicians, physicians, and engineers since the early 1990s. Fractional calculus is a branch of mathematical analysis that focuses on the study of differential operators of arbitrary order. Fractional integrals and derivatives extend the well-known definitions of integerorder primitives and derivatives to the ordinary differential calculus to real-order operators. Abel was the first to attack a physical problem using the techniques of fractional calculus. The increased interest in this field is due to the fact that the fractional differential operators are nonlocal and therefore enable us to provide better description of real phenomena. A brief history of the development of fractional calculus can be found in Ross [18] and Miller and Ross [19]. A survey of many emerging applications of the fractional calculus in the area of science and engineering is done in the text by Podlubny [20].

A quasi-static uncoupled theory of thermoelasticity based on fractional heat conduction equation was constructed by Povstenko [21]. The Caputo time-fractional derivative [22] was used by Povstenko [23] to investigate thermal stresses in an infinite body with a circular cylindrical hole. Sherief et al. [24] put forward a new model of generalized thermoelasticity using fractional calculus. A uniqueness theorem, reciprocity relation, and variational principle have also been established in the same article. Youssef [25] also proposed a new theory of generalized thermoelasticity using the methodology of fractional calculus and discussed one-dimensional application. Deswal and Kalkal [26] employed state space approach to study the magneto-thermoelastic interactions in an initially stressed isotropic medium under the purview of two-temperature theory of generalized thermoelasticity. The problem of magnetothermoelastic interactions in an unbounded and perfectly conducting half-space whose surface suffers from a time harmonic thermal shock in the context of micropolar generalized thermoelasticity with fractional heat transfer has been analyzed by Deswal and Kalkal [27]. Abbas [28] considered the problem of fractional order thermoelastic interaction in a material placed in a magnetic field and subjected to moving plane heat source. Santra et al. [29] aimed at studying the effect of rotation on thermoelastic interactions in a homogeneous isotropic three-dimensional medium whose surface is traction-free and is subjected to a time-dependent heat source. The problem has been modelled on the basis of fractional order generalized thermoelasticity. Wang et al. [30] suggested a new theory of generalized thermoelasticity for elastic media with variable properties in the context of fractional order heat conduction equation. They derived the formulations of anisotropic heterogeneous material with temperature dependent material properties by making use of the Clausius inequality and the higher expansions of free energy.

Recently, Youssef [31] derived a new theory of thermoelasticity by modifying the Duhamel-Neumann stressstrain relation. In this theory, this relation depends on the fractional order of strain which adds knowledge about the time history to the deformation of materials after being acted upon by mechanical or thermal loadings. In his work, Youssef constructed a new unified system of differential equations governing seven different models of thermoelasticity in the context of one-temperature type and two-temperature type. A one-dimensional application of the thermoelasticity with fractional order strain for an isotropic and homogeneous medium for some models is also elaborated in the same article.

Due to the extensive engineering applications, such as pulsed-laser cutting and welding and high speed machining and grinding, several research works have been devoted to problems involving a moving heat source or thermal shock. Danilovskaya [32] was the first who solved a dynamical heat source problem under the purview of coupled thermoelasticity. The problems of instantaneous and moving heat sources in infinite and semi-infinite space and static line heat sources in semi-infinite space were considered by Eason and Sneddon [33], Nowacki [34], and others under the coupled theory of thermoelasticity. Sherief and Anwar [35] investigated the thermoelastic interactions due to a continuous line heat source in a linear, homogeneous unbounded solid in the context of the Lord-Shulman model of generalized thermoelasticity.

The theoretical study and applications in viscoelastic materials have become an important task for solid mechanics with the rapid development of polymer science and plastic industry as well as with the wide use of materials under high temperature in modern technology and application of biology and geology in engineering. Freudenthal [36] commented that most solids, when subjected to loading, exhibit viscous effects, particularly at an elevated temperature. The stress-strain law for many materials such as polycrystalline metals and high polymers can be approximated by the linear viscoelasticity theory. So the consideration of viscoelastic properties of the medium makes the studies more meaningful. The theory of thermoviscoelasticity and the solutions of some boundary value problems of thermoviscoelasticity 
were explored by Ilioushin and Pobedria [37]. Several investigations relating to thermoviscoelasticity theory have been presented in [38-42].

In the present work, a viscoelastic medium with internal heat source is considered. The governing equations are taken in the context of GN-III model of two-temperature generalized thermoelasticity with fractional order strain. Laplace transform and state-space techniques are adopted to find out the general solution of the problem. The results obtained theoretically have been computed numerically and depicted graphically. Some comparisons are exhibited in figures to demonstrate the effects of fractional order strain, viscosity, two-temperature parameter, and the presence of internal heat source.

\section{Basic Equations and Problem Formulation}

Following Youssef [31], the constitutive equations and the field equations for generalized viscoelastic two-temperature GN-III model of thermoelasticity with fractional order strain and in the presence of internal heat source may be written as follows:

(i) The constitutive relation is

$$
\begin{aligned}
\sigma_{i j}= & 2 \mu^{*}\left(1+\tau^{\beta} D_{t}^{\beta}\right) e_{i j}+\lambda^{*}\left(1+\tau^{\beta} D_{t}^{\beta}\right) e_{k k} \delta_{i j} \\
& -\beta_{1}^{*} \theta \delta_{i j}, \quad(i, j=1,2,3) .
\end{aligned}
$$

(ii) The strain-displacement relation is

$$
e_{i j}=\frac{1}{2}\left(u_{i, j}+u_{j, i}\right) \text {. }
$$

(iii) Equation of motion is

$$
\sigma_{j i, j}=\rho \ddot{u}_{i}
$$

(iv) Two-temperature heat conduction equation is

$$
K^{*} \varphi_{, i i}+k \dot{\varphi}_{, i i}=\rho c_{E} \ddot{\theta}+\beta_{1}^{*} T_{0}\left(1+\tau^{\beta} D_{t}^{\beta}\right) \ddot{e}-\dot{Q} .
$$

(v) Relation between thermodynamical and conductive temperature is

$$
\varphi-\theta=a \varphi_{, i i}
$$

In the preceding equations, $\sigma_{i j}$ and $e_{i j}$ are the components of stress and strain tensors, respectively, $u_{i}(i=x, y, z)$ are the components of displacement vector, $\theta=T-T_{0}$ represents the thermodynamical temperature, $T$ being the absolute temperature and $T_{0}$ denoting the temperature of medium in its natural state, $\varphi=\varphi-T_{0}$ stands for the conductive temperature, $\rho$ is the density of medium, $\tau$ is mechanical relaxation time, $\beta$ is the fractional strain parameter, $\lambda^{*}=$ $\lambda_{e}\left(1+\alpha_{0}(\partial / \partial t)\right), \mu^{*}=\mu_{e}\left(1+\alpha_{1}(\partial / \partial t)\right), \beta_{1}^{*}=\beta_{1 e}(1+$ $\left.\beta_{1}(\partial / \partial t)\right), \beta_{1 e}=\left(3 \lambda_{e}+2 \mu_{e}\right) \alpha_{t}, \beta_{1}=\left(3 \lambda_{e} \alpha_{0}+2 \mu_{e} \alpha_{1}\right) \alpha_{t} / \beta_{1 e}$, $\lambda_{e}$ and $\mu_{e}$ are Lame's elastic constants, $\alpha_{0}$ and $\alpha_{1}$ are the viscoelastic relaxation times, $\alpha_{t}$ is the coefficient of linear thermal expansion, $c_{E}$ represents specific heat at constant strain, $k$ is thermal conductivity, $K^{*}=c_{E}\left(\lambda_{e}+2 \mu_{e}\right) / 4$ stands for the material characteristic of GN theory, $Q$ is the heat source, a dot over a variable denotes derivative with respect to time $t$, a comma refers to a spatial derivative, and the tensor convention of summing over repeated indices is used.

By taking $\tau=0$ in the above governing equations, we may resume the equations of two-temperature thermoviscoelasticity with internal heat source in the context of GN-III theory.

We consider an infinite thermoelastic solid body which is unstrained and unstressed initially at uniform temperature $T_{0}$ occupying the region $-\infty<x<\infty$, whose state depends only on the space variable $x$ and time $t$ so that the displacement vector $\vec{u}$, conductive temperature $\varphi$, and thermodynamical temperature $\theta$ can be expressed in the following form:

$$
\begin{aligned}
& u_{x}=u(x, t), \\
& u_{y}=0, \\
& u_{z}=0, \\
& \varphi=\varphi(x, t), \\
& \theta=\theta(x, t) .
\end{aligned}
$$

The governing equations (1)-(5) in one-dimensional case assume the shape

$$
\begin{aligned}
\sigma & =\sigma_{x x} \\
& =\left(\lambda^{*}+2 \mu^{*}\right)\left(1+\tau^{\beta} D_{t}^{\beta}\right) e-\beta_{1}^{*} \theta, \\
e & =e_{x x}=\frac{\partial u}{\partial x}, \\
\frac{\partial \sigma}{\partial x} & =\rho \ddot{u}, \\
K^{*} \frac{\partial^{2} \varphi}{\partial x^{2}}+k \frac{\partial^{3} \varphi}{\partial t \partial x^{2}} & =\rho c_{E} \frac{\partial^{2} \theta}{\partial t^{2}}+\beta_{1}^{*} T_{0}\left(1+\tau^{\beta} D_{t}^{\beta}\right) \ddot{e} \\
& -\dot{Q}, \\
\varphi-\theta & =a \frac{\partial^{2} \varphi}{\partial x^{2}} .
\end{aligned}
$$

Equation (9) may also be expressed as

$$
\frac{\partial^{2} \sigma}{\partial x^{2}}=\rho \ddot{e} .
$$

Now we transform the above equations into nondimensional forms by introducing the following dimensionless parameters:

$$
\begin{aligned}
x^{\prime} & =\frac{\bar{\omega}}{c_{1}} x, \\
u^{\prime} & =\frac{\rho c_{1} \bar{\omega}}{\beta_{1 e} T_{0}} u, \\
\left(t^{\prime}, \tau^{\prime}, \alpha_{0}^{\prime}, \alpha_{1}^{\prime}, \beta_{1}^{\prime}\right) & =\bar{\omega}\left(t, \tau, \alpha_{0}, \alpha_{1}, \beta_{1}\right),
\end{aligned}
$$




$$
\begin{aligned}
\theta^{\prime} & =\frac{\theta}{T_{0}}, \\
\varphi^{\prime} & =\frac{\varphi}{T_{0}}, \\
\sigma^{\prime} & =\frac{1}{\beta_{1 e} T_{0}} \sigma, \\
Q^{\prime} & =\frac{Q}{\rho c_{E} T_{0} \bar{\omega}},
\end{aligned}
$$

where $\bar{\omega}=c_{E}\left(\lambda_{e}+2 \mu_{e}\right) / k$ and $c_{1}^{2}=\left(\lambda_{e}+2 \mu_{e}\right) / \rho$.

Equations (7) and (10)-(12) may now be reduced to the following system of dimensionless equations (after removing the primes for clarity):

$$
\begin{aligned}
\sigma= & \left(1+\delta_{0} \frac{\partial}{\partial t}\right)\left(1+\tau^{\beta} D_{t}^{\beta}\right) e \\
& -\left(1+\beta_{1} \frac{\partial}{\partial t}\right) \theta \\
\left(1+\epsilon \frac{\partial}{\partial t}\right) \frac{\partial^{2} \varphi}{\partial x^{2}}= & \epsilon \frac{\partial^{2} \theta}{\partial t^{2}} \\
& +\zeta\left(1+\beta_{1} \frac{\partial}{\partial t}\right)\left(1+\tau^{\beta} D_{t}^{\beta}\right) \frac{\partial^{2} e}{\partial t^{2}} \\
& -\epsilon \frac{\partial Q}{\partial t}, \\
\varphi-\theta= & \alpha \frac{\partial^{2} \varphi}{\partial x^{2}}, \\
\frac{\partial^{2} \sigma}{\partial x^{2}}= & \frac{\partial^{2} e}{\partial t^{2}}
\end{aligned}
$$

where $\delta_{0}=\left(\lambda_{e} \alpha_{0}+2 \mu_{e} \alpha_{1}\right) / \rho c_{1}^{2}, \epsilon=\bar{\omega} k / K^{*}$, and $\zeta=$ $\beta_{1 e}^{2} T_{0} / \rho K^{*}$ are the coupling parameters and $\alpha=a \bar{\omega}^{2} / c_{1}^{2}$ is the temperature discrepancy.

Performing the Laplace transform defined as

$$
\bar{f}(x, s)=L\{f(x, t)\}=\int_{0}^{\infty} f(t) e^{-s t} d t,
$$

over (14)-(17), and using the homogeneous initial conditions, we obtain the following system of differential equations:

$$
\begin{aligned}
\bar{\sigma} & =\left(1+\delta_{0} s\right)\left(1+\tau^{\beta} s^{\beta}\right) \bar{e}-\left(1+\beta_{1} s\right) \bar{\theta} \\
(1+\epsilon s) D^{2} \bar{\varphi}= & \epsilon s^{2} \bar{\theta}+\zeta\left(1+\beta_{1} s\right)\left(1+\tau^{\beta} s^{\beta}\right) s^{2} \bar{e} \\
-\epsilon s \bar{Q} & \\
\bar{\varphi}-\bar{\theta} & =\alpha D^{2} \bar{\varphi}, \\
D^{2} \bar{\sigma} & =s^{2} \bar{e},
\end{aligned}
$$

where $D \equiv \partial / \partial x$.
We consider that a moving heat source of constant strength is located at the origin and at time $t=0^{+}$begins moving along the positive direction of $x$-axis with a constant velocity $v$ releasing its energy continuously. This moving heat source is considered to be of the following nondimensional form:

$$
Q=Q_{0} \delta(x-v t) H(t)
$$

where $Q_{0}$ is the strength of the heat source, $\delta(x)$ is wellknown Dirac-delta function, and $H(t)$ stands for Heaviside unit step function. Applying Laplace transform defined in (18) and Fourier transform defined as

$$
f^{*}(\xi, s)=\int_{-\infty}^{\infty} \bar{f}(x, s) e^{-\imath \xi x} d x
$$

to (20) and then inverting Fourier transform manually, we get

$$
\bar{Q}=\frac{Q_{0}}{2 v} e^{(\iota s / v) x}[2 H(x)-1]
$$

As in the current problem, the heat source is moving along the positive $x$-axis, and therefore we have

$$
\bar{Q}=\frac{Q_{0}}{2 v} e^{(\iota s / v) x} .
$$

Eliminating $\bar{e}$ and $\bar{\theta}$ from (19), we arrive at the following system of differential equations:

$$
\begin{aligned}
& D^{2} \bar{\varphi}=L_{1} \bar{\varphi}+L_{2} \bar{\sigma}+L_{3}, \\
& D^{2} \bar{\sigma}=M_{1} \bar{\varphi}+M_{2} \bar{\sigma}+M_{3},
\end{aligned}
$$

where

$$
\begin{aligned}
& L_{1}=\frac{s^{2}\left\{\epsilon\left(1+\delta_{0} s\right)+\zeta\left(1+\beta_{1} s\right)^{2}\right\}}{\left(1+\delta_{0} s\right)(1+\epsilon s(1+\alpha s))+\alpha \zeta s^{2}\left(1+\beta_{1} s\right)^{2}}, \\
& L_{2}=\frac{\zeta s^{2}\left(1+\beta_{1} s\right)}{\left(1+\delta_{0} s\right)(1+\epsilon s(1+\alpha s))+\alpha \zeta s^{2}\left(1+\beta_{1} s\right)^{2}},
\end{aligned}
$$

$L_{3}$

$$
\begin{aligned}
&=- \frac{\epsilon s\left(1+\delta_{0} s\right) Q_{0} e^{(l s / v) x}}{v\left[\left(1+\delta_{0} s\right)(1+\epsilon s(1+\alpha s))+\alpha \zeta s^{2}\left(1+\beta_{1} s\right)^{2}\right]}, \\
& M_{1}=\frac{s^{2}\left(1+\beta_{1} s\right)\left(1-\alpha L_{1}\right)}{\left(1+\delta_{0} s\right)\left(1+\tau^{\beta} s^{\beta}\right)}, \\
& M_{2}=\frac{s^{2}\left[1-\alpha L_{2}\left(1+\beta_{1} s\right)\right]}{\left(1+\delta_{0} s\right)\left(1+\tau^{\beta} s^{\beta}\right)} \\
& M_{3}=-\frac{L_{3} \alpha s^{2}\left(1+\beta_{1} s\right)}{\left(1+\delta_{0} s\right)\left(1+\tau^{\beta} s^{\beta}\right)} .
\end{aligned}
$$




\section{State-Space Formulation}

Having chosen the conductive temperature $\bar{\varphi}$ and stress component $\bar{\sigma}$ as state variables, (24) may be recast in matrix form as

$$
D^{2} \bar{V}(x, s)=A(s) \bar{V}(x, s)+B(x, s),
$$

where

$$
\begin{aligned}
\bar{V}(x, s) & =\left[\begin{array}{l}
\bar{\varphi}(x, s) \\
\bar{\sigma}(x, s)
\end{array}\right], \\
A(s) & =\left[\begin{array}{ll}
L_{1} & L_{2} \\
M_{1} & M_{2}
\end{array}\right], \\
B(x, s) & =\left[\begin{array}{l}
L_{3} \\
M_{3}
\end{array}\right] .
\end{aligned}
$$

The formal solution of the differential equation (26) may be written as

$$
\begin{aligned}
\bar{V}(x, s)= & \exp [-\sqrt{A(s)} x] \\
& \cdot\left[\bar{V}(0, s)+\left\{A(s)+\frac{s^{2}}{v^{2}} I\right\}^{-1} B(0, s)\right] \\
& -\left[A(s)+\frac{s^{2}}{v^{2}} I\right]^{-1} B(x, s),
\end{aligned}
$$

where $\bar{V}(0, s)=\left[\begin{array}{l}\bar{\varphi}(0, s) \\ \bar{\sigma}(0, s)\end{array}\right]=\left[\begin{array}{c}\bar{\varphi}_{0} \\ \bar{\sigma}_{0}\end{array}\right]$ and $I$ is an identity matrix of second order. The terms containing exponents of growing nature in the space variable $x$ have been discarded due to the regularity condition at infinity.

If there is no heat source inside the medium, then (28) assumes the form

$$
\bar{V}(x, s)=\exp [-\sqrt{A(s)} x] \bar{V}(0, s) .
$$

The characteristic equation of matrix $A(s)$ is obtained as

$$
\lambda^{2}-\left(L_{1}+M_{2}\right) \lambda+L_{1} M_{2}-L_{2} M_{1}=0,
$$

where the roots $\lambda_{1}$ and $\lambda_{2}$ of (30) must satisfy

$$
\begin{aligned}
\lambda_{1}+\lambda_{2} & =L_{1}+M_{2}, \\
\lambda_{1} \lambda_{2} & =L_{1} M_{2}-L_{2} M_{1} .
\end{aligned}
$$

The Taylor series expansion of the matrix exponential has the form

$$
\exp [-\sqrt{A(s)} x]=\sum_{n=0}^{\infty} \frac{[-\sqrt{A(s)} x]^{n}}{n !}
$$

Making use of the well-known Cayley-Hamilton theorem, we can express $A^{2}$ and higher orders of matrix $A$ in terms of $I$ and A.
Thus the infinite series in (32) can be truncated as

$$
\exp [-\sqrt{A(s)} x]=a_{0}(x, s) I+a_{1}(x, s) A
$$

where $a_{0}$ and $a_{1}$ are constants depending on $x$ and $s$.

Again by Cayley-Hamilton theorem the characteristic roots $\lambda_{1}$ and $\lambda_{2}$ of the matrix $A$ must satisfy (33). Therefore, we have

$$
\begin{aligned}
& \exp \left[-\sqrt{\lambda_{1}} x\right]=a_{0}+a_{1} \lambda_{1}, \\
& \exp \left[-\sqrt{\lambda_{2}} x\right]=a_{0}+a_{1} \lambda_{2} .
\end{aligned}
$$

On solving the above linear system of equations, we obtain

$$
\begin{aligned}
& a_{0}=\frac{\lambda_{1} e^{-\sqrt{\lambda_{2}} x}-\lambda_{2} e^{-\sqrt{\lambda_{1}} x}}{\lambda_{1}-\lambda_{2}}, \\
& a_{1}=\frac{e^{-\sqrt{\lambda_{1}} x}-e^{-\sqrt{\lambda_{2}} x}}{\lambda_{1}-\lambda_{2}} .
\end{aligned}
$$

Substituting the values of $a_{0}$ and $a_{1}$ along with $I$ and $A$ into (33), we have

$$
\exp [-\sqrt{A(s)} x]=\Gamma_{i j}(x, s), \quad(i, j=1,2),
$$

where the components $\Gamma_{i j}(x, s)$ are given by

$$
\begin{aligned}
& \Gamma_{11}=\frac{e^{-\sqrt{\lambda_{2}} x}\left(\lambda_{1}-L_{1}\right)-e^{-\sqrt{\lambda_{1}} x}\left(\lambda_{2}-L_{1}\right)}{\lambda_{1}-\lambda_{2}}, \\
& \Gamma_{12}=\frac{L_{2}\left(e^{-\sqrt{\lambda_{1}} x}-e^{-\sqrt{\lambda_{2}} x}\right)}{\lambda_{1}-\lambda_{2}}, \\
& \Gamma_{21}=\frac{M_{1}\left(e^{-\sqrt{\lambda_{1}} x}-e^{-\sqrt{\lambda_{2}} x}\right)}{\lambda_{1}-\lambda_{2}}, \\
& \Gamma_{22}=\frac{e^{-\sqrt{\lambda_{2}} x}\left(\lambda_{1}-M_{2}\right)-e^{-\sqrt{\lambda_{1}} x}\left(\lambda_{2}-M_{2}\right)}{\lambda_{1}-\lambda_{2}} .
\end{aligned}
$$

Hence solution (28) can be written as

$$
\begin{aligned}
\bar{V}(x, s)= & \Gamma_{i j}\left[\bar{V}(0, s)+\left\{A(s)+\frac{s^{2}}{v^{2}} I\right\}^{-1} B(0, s)\right] \\
& -\left[A(s)+\frac{s^{2}}{v^{2}} I\right]^{-1} B(x, s) .
\end{aligned}
$$


Plugging the values of $\bar{V}(x, s),\left[A(s)+\left(s^{2} / v^{2}\right) I\right]^{-1}$, and $B(x, s)$ into (38) and after some straightforward calculation, the expressions for conductive temperature and stress are evaluated as

$$
\begin{aligned}
& \bar{\varphi}(x, s)=\frac{1}{\lambda_{1}-\lambda_{2}}\left[\left(\lambda_{1} \bar{\varphi}_{0}-L_{1} \bar{\varphi}_{0}-L_{2} \bar{\sigma}_{0}+\gamma_{1} \lambda_{1}\right.\right. \\
& \left.-\gamma_{1} L_{1}-\gamma_{2} L_{2}\right) e^{-\sqrt{\lambda_{2}} x}-\left(\lambda_{2} \bar{\varphi}_{0}-L_{1} \bar{\varphi}_{0}-L_{2} \bar{\sigma}_{0}\right. \\
& \left.\left.+\gamma_{1} \lambda_{2}-\gamma_{1} L_{1}-\gamma_{2} L_{2}\right) e^{-\sqrt{\lambda_{1}} x}\right]-\gamma_{3}, \\
& \bar{\sigma}(x, s)=\frac{1}{\lambda_{1}-\lambda_{2}}\left[\left(\lambda_{1} \bar{\sigma}_{0}-M_{2} \bar{\sigma}_{0}-M_{1} \bar{\varphi}_{0}-\gamma_{1} M_{1}\right.\right. \\
& \left.+\lambda_{1} \gamma_{2}-M_{2} \gamma_{2}\right) e^{-\sqrt{\lambda_{2}} x}+\left(M_{2} \bar{\sigma}_{0}-\lambda_{2} \bar{\sigma}_{0}+M_{1} \bar{\varphi}_{0}\right. \\
& \left.\left.+\gamma_{1} M_{1}-\lambda_{2} \gamma_{2}+M_{2} \gamma_{2}\right) e^{-\sqrt{\lambda_{1}} x}\right]-\gamma_{4},
\end{aligned}
$$

where

$$
\begin{aligned}
& \gamma_{1}=\frac{T_{2}}{T_{1}} \\
& \gamma_{2}=\frac{T_{3}}{T_{1}} \\
& \gamma_{3}=\frac{T_{4}}{T_{1}} \\
& \gamma_{4}=\frac{T_{5}}{T_{1}} \\
& T_{1}=\frac{s^{4}}{v^{4}}+\frac{s^{2}}{v^{2}}\left(L_{1}+M_{2}\right)+L_{1} M_{2}-L_{2} M_{1}, \\
& T_{2}=N_{1} M_{2}-L_{2} N_{2}+\frac{s^{2}}{v^{2}} N_{1} \\
& T_{3}=L_{1} N_{2}-M_{1} N_{1}+\frac{s^{2}}{v^{2}} N_{2} \\
& T_{4}=L_{3} M_{2}-L_{2} M_{3}+\frac{s^{2}}{v^{2}} L_{3}, \\
& T_{5}=L_{1} M_{3}-L_{3} M_{1}+\frac{s^{2}}{v^{2}} M_{3}, \\
& N_{1} \\
& =\frac{-\epsilon s\left(1+\delta_{0} s\right) Q_{0}}{\nu\left[\left(1+\delta_{0} s\right)\{1+\epsilon s(1+\alpha s)\}+\alpha \zeta s^{2}\left(1+\beta_{1} s\right)^{2}\right]}, \\
& N_{2}=\frac{-N_{1} \alpha s^{2}\left(1+\beta_{1} s\right)}{\left(1+\delta_{0} s\right)\left(1+\tau^{\beta} s^{\beta}\right)} .
\end{aligned}
$$

4.2. Thermal Boundary Condition. The medium at $x=0$ is kept at reference temperature $T_{0}$; that is,

$$
\varphi(0, t)=\varphi_{0}=0 .
$$

Operating Laplace transform on the above equation, one can obtain

$$
\varphi(0, s)=\bar{\varphi}_{0}=0 .
$$

Hence, we can utilize the values of $\bar{\sigma}_{0}$ and $\bar{\varphi}_{0}$ from (43) and (45) in (39) and (41) to finally achieve the solutions in the Laplace transform domain as

$$
\begin{aligned}
\bar{\varphi} & =\frac{1}{\lambda_{1}-\lambda_{2}}\left[\left\{\gamma_{1}\left(\lambda_{1}-L_{1}\right)+L_{2}\left(\frac{\sigma^{*}}{s}-\gamma_{2}\right)\right\} e^{-\sqrt{\lambda_{2}} x}\right. \\
& \left.-\left\{\gamma_{1}\left(\lambda_{2}-L_{1}\right)+L_{2}\left(\frac{\sigma^{*}}{s}-\gamma_{2}\right)\right\} e^{-\sqrt{\lambda_{1}} x}\right]-\gamma_{3},
\end{aligned}
$$




$$
\begin{aligned}
\bar{\sigma} & =\frac{1}{\lambda_{1}-\lambda_{2}}\left[\left\{\left(\lambda_{1}-M_{2}\right)\left(\gamma_{2}-\frac{\sigma^{*}}{s}\right)-\gamma_{1} M_{1}\right\}\right. \\
& \cdot e^{-\sqrt{\lambda_{2}} x}-\left\{\left(\lambda_{2}-M_{2}\right)\left(\gamma_{2}-\frac{\sigma^{*}}{s}\right)-\gamma_{1} M_{1}\right\} \\
& \left.\cdot e^{-\sqrt{\lambda_{1}} x}\right]-\gamma_{4}, \\
\bar{\theta} & =\frac{1}{\lambda_{1}-\lambda_{2}}\left[\left\{\gamma_{1}\left(\lambda_{1}-L_{1}\right)+L_{2}\left(\frac{\sigma^{*}}{s}-\gamma_{2}\right)\right\}\right. \\
& \cdot\left(1-\alpha \lambda_{2}\right) e^{-\sqrt{\lambda_{2}} x} \\
& -\left\{\gamma_{1}\left(\lambda_{2}-L_{1}\right)+L_{2}\left(\frac{\sigma^{*}}{s}-\gamma_{2}\right)\right\}\left(1-\alpha \lambda_{1}\right) \\
& \left.\cdot e^{-\sqrt{\lambda_{1}} x}\right]-\gamma_{3}\left(1+\alpha \frac{s^{2}}{v^{2}}\right) .
\end{aligned}
$$

Using dimensionless variables and Laplace transform in (9), the displacement component may be evaluated as

$$
\bar{u}=\frac{1}{\alpha_{1} s^{2}} \frac{\partial \bar{\sigma}}{\partial x}
$$

where $\alpha_{1}=\beta_{1 e} T_{0} / \rho c_{1}^{2}$.

Substitution of $\bar{\sigma}$ from (47) into the above equation yields

$$
\begin{aligned}
\bar{u} & =\frac{-1}{\alpha_{1} s^{2}\left(\lambda_{1}-\lambda_{2}\right)}\left[\sqrt { \lambda _ { 2 } } \left\{\left(\lambda_{1}-M_{2}\right)\left(\gamma_{2}-\frac{\sigma^{*}}{s}\right)\right.\right. \\
& \left.-\gamma_{1} M_{1}\right\} e^{-\sqrt{\lambda_{2}} x}-\sqrt{\lambda_{1}}\left\{\left(\lambda_{2}-M_{2}\right)\left(\gamma_{2}-\frac{\sigma^{*}}{s}\right)\right. \\
& \left.\left.-\gamma_{1} M_{1}\right\} e^{-\sqrt{\lambda_{1}} x}\right] .
\end{aligned}
$$

\section{Limiting Cases}

5.1. Without Viscous Effect. If we neglect the effect of viscosity, then we will be left with the corresponding problem in generalized two-temperature thermoelasticity with fractional order strain. In this case, we put $\alpha_{0}=\alpha_{1}=0$ which implies $\lambda^{*}=\lambda_{e}, \mu^{*}=\mu_{e}, \beta_{1}^{*}=\beta_{1 e}$, and $\beta_{1}=0$. By implementing the above changes, the corresponding expressions of the physical fields can be obtained from (46)-(48) and (50).

5.2. With One Temperature. By setting $a=0$ and consequently $\alpha=0$ in the governing equations, we get the expressions for different field variables from (46)-(48) and (50) for one-temperature case, that is, the case when conductive temperature coincides with thermodynamical temperature.

5.3. Without Internal Heat Source. Neglecting the influence of internal heat source, that is, $\left(Q_{0}=0\right)$, the expressions of conductive temperature, stress, thermodynamical temperature, and displacement are obtained in a viscothermoelastic medium with two-temperature and fractional order strain as

$$
\begin{aligned}
\bar{\varphi}= & \frac{L_{2} \sigma^{*}}{s\left(\lambda_{1}-\lambda_{2}\right)}\left(e^{-\sqrt{\lambda_{2}} x}-e^{-\sqrt{\lambda_{1}} x}\right), \\
\bar{\sigma}= & \frac{\sigma^{*}}{s\left(\lambda_{1}-\lambda_{2}\right)}\left[e^{-\sqrt{\lambda_{1}} x}\left(\lambda_{2}-M_{2}\right)\right. \\
& \left.-e^{-\sqrt{\lambda_{2}} x}\left(\lambda_{1}-M_{2}\right)\right], \\
\bar{\theta}= & \frac{L_{2} \sigma^{*}}{s\left(\lambda_{1}-\lambda_{2}\right)}\left[\left(1-\alpha \lambda_{2}\right) e^{-\sqrt{\lambda_{2}} x}\right. \\
& \left.-\left(1-\alpha \lambda_{1}\right) e^{-\sqrt{\lambda_{1}} x}\right], \\
\bar{u}= & \frac{\sigma^{*}}{\alpha_{1} s^{3}\left(\lambda_{1}-\lambda_{2}\right)}\left[\sqrt{\lambda_{2}}\left(\lambda_{1}-M_{2}\right) e^{-\sqrt{\lambda_{2}} x}\right. \\
& \left.-\sqrt{\lambda_{1}}\left(\lambda_{2}-M_{2}\right) e^{-\sqrt{\lambda_{1}} x}\right] .
\end{aligned}
$$

\section{Numerical Inversion of the Transform}

Equations (46)-(48) and (50) provide the expressions for conductive temperature, stress, thermodynamical temperature, and displacement in Laplace transform domain. To determine these in physical domain, Laplace inversion is applied with the help of numerical technique based on Fourier expansion of functions performed by Honig and Hirdes [43].

Let $\bar{f}(s)$ be the Laplace transform of function $f(t)$. The inversion formula of Laplace transform states that

$$
f(t)=\frac{1}{2 \pi \iota} \int_{d-\iota \infty}^{d+\iota \infty} e^{s t} \bar{f}(s) d s
$$

where $d$ is an arbitrary real number greater than all the real parts of singularities of $\bar{f}(s)$. Taking $s=d+\imath y$ and using Fourier series in the interval $[0,2 T]$, we get the approximate formula

$$
f(t) \cong f_{N}(t)=\frac{1}{2} c_{0}+\sum_{k=1}^{N} c_{k}, \quad \text { for } 0 \leq t \leq 2 T,
$$

where

$$
c_{k}=\frac{e^{d t}}{T} \operatorname{Re}\left[e^{\iota k \pi t / T} \bar{f}\left(d+\frac{\iota k \pi}{T}\right)\right]
$$

and $N$ is a sufficiently large integer representing the number of terms in the truncated Fourier series, chosen such that

$$
e^{d t} \operatorname{Re}\left[e^{\iota N \pi t / T} \bar{f}\left(d+\frac{\iota N \pi}{T}\right)\right] \leq \epsilon_{1}
$$

where $\epsilon_{1}$ is a prescribed small positive value that corresponds to the degree of accuracy to be achieved. 


\section{Numerical Results and Discussion}

With an aim of illustrating the contribution of fractional strain parameter, mechanical relaxation time, twotemperature parameter, viscosity coefficients, and heat source on field quantities, a numerical analysis is carried out. For this purpose, we have taken the following values of relevant parameters:

$$
\begin{aligned}
& \lambda_{e}=7.76 \times 10^{10} \mathrm{~kg} \mathrm{~m}^{-1} \mathrm{~s}^{-2}, \\
& \mu_{e}=3.86 \times 10^{10} \mathrm{~kg} \mathrm{~m}^{-1} \mathrm{~s}^{-2}, \\
& \rho=8954 \mathrm{~kg} \mathrm{~m}^{-3} \text {, } \\
& c_{E}=383.1 \mathrm{~J} \mathrm{~kg}^{-1} \mathrm{~K}^{-1} \text {, } \\
& \alpha_{t}=1.78 \times 10^{-5} \mathrm{~K}^{-1}, \\
& k=386 \mathrm{~W} \mathrm{~m}^{-1} \mathrm{~K}^{-1} \text {, } \\
& \alpha_{0}=0.6 \mathrm{~s} \text {, } \\
& \alpha_{1}=0.9 \mathrm{~s} \text {, } \\
& t=0.1 \mathrm{~s} \text {, } \\
& \tau=0.01 \mathrm{~s}, \\
& \sigma^{*}=1 \text {, } \\
& Q_{0}=2 \text {, } \\
& \alpha=0.1, \\
& v=0.5 \text {, } \\
& \beta=0.5 \text {. }
\end{aligned}
$$

Making use of the above mentioned numerical values, we have computed the dimensionless values of displacement, stress, thermodynamical temperature, and conductive temperature with distance $x$ and shown them graphically in four groups. In the first group (Figures 1-4), we have shown the effects of mechanical relaxation time $\tau$ and parameter $\beta$ on the considered physical variables with location $x$. For this, three different sets of values of $\tau$ and $\beta$ are considered: (i) $\tau=0$ and $\beta \neq 0$, (ii) $\tau=0.01$ and $\beta=0.5$, and (iii) $\tau=0.01$ and $\beta=1.0$. The case $\tau=0$ leads to GreenNaghdi model of type III while $\tau=0.01$ leads to the same model in context of fractional order strain. The influence of the velocity of internal heat source on the considered physical variables for three values of $v$, namely, $0.1,0.5$, and 1.0 , is shown graphically in the second group (Figures 5-8). Attention is paid to the investigation of effects of viscosity and two-temperature parameter on the physical quantities in the third group (Figures 9-12). For this, all the considered field variables are examined for three different cases: (i) thermoviscoelastic solid with two-temperature under fractional order strain and heat source (TV2T), (ii) thermoelastic solid with two-temperature under fractional order strain and heat source (T2T), and (iii) thermoviscoelastic solid with one temperature under fractional order strain and heat source

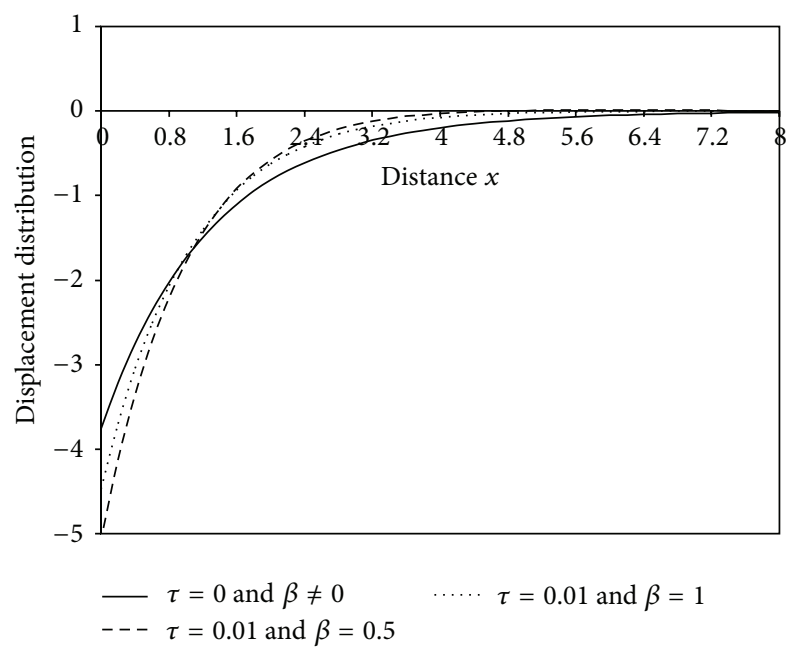

Figure 1: Profile of displacement distribution.

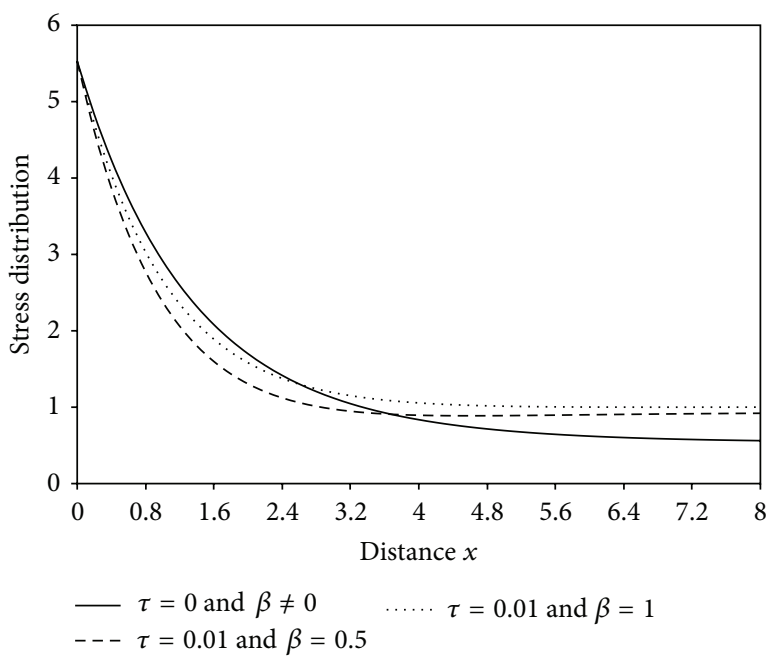

Figure 2: Profile of stress distribution.

(TV1T). Pattern of different fields in the presence and absence of heat source has been observed in the fourth group (Figures 13-16).

Group I. Figure 1 elucidates the variations of displacement field with distance $x$. Displacement field starts with its maximum numerical value for all the considered values of $\tau$ and $\beta$ and then diminishes to zero with the passage of time. Significant differences in the values of displacement for the presence $(\tau=0.01)$ and absence $(\tau=0)$ of mechanical relaxation time can be noticed from the figure. The values of displacement field for a particular range exhibit sufficient difference for two values of parameter $\beta$. This distinction decreases with the increase of distance from the point of application of source.

In Figure 2, we have shown the profile of stress distribution to study the effects of mechanical relaxation time $\tau$ and parameter $\beta$. Stress field enjoys a similar trend of variations for all the considered values of $\tau$ and $\beta$; however dissimilarity 


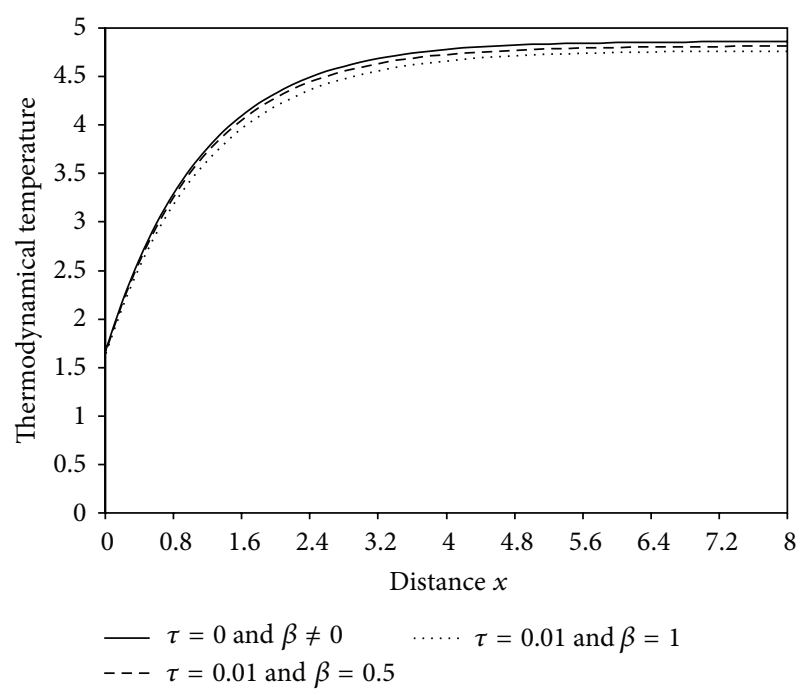

FIGURE 3: Profile of thermodynamical temperature distribution.

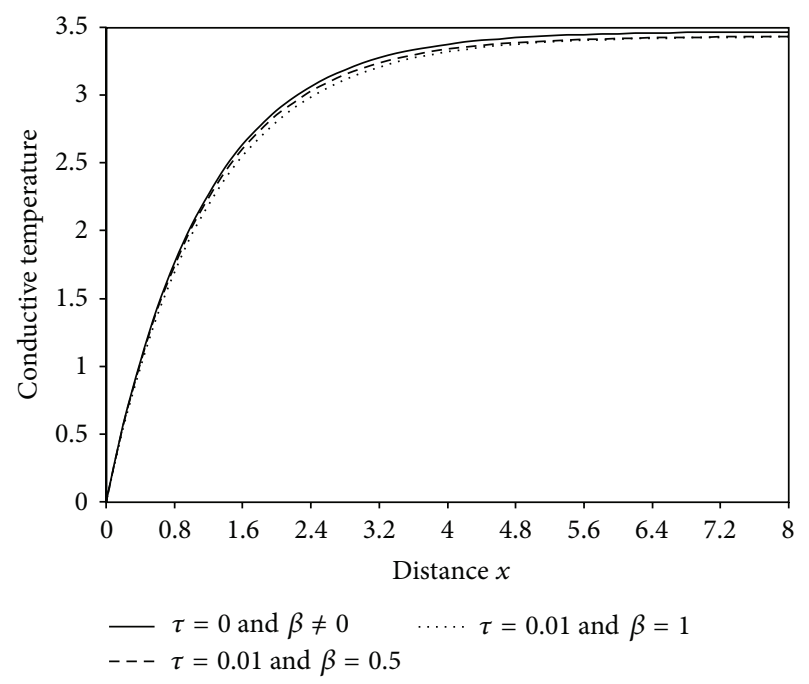

FIGURE 4: Profile of conductive temperature distribution.

lies on the grounds of magnitude. Stress field shows significant sensitivity towards the mechanical relaxation time. The figure also reveals the pronounced effects of parameter $\beta$ on the stress distribution.

The effects of relaxation time $\tau$ and fractional strain parameter $\beta$ on thermodynamical temperature distribution with nondimensional location $x$ are depicted in Figure 3 . The thermodynamical temperature experiences a similar pattern of variations for all the three cases having differences in magnitude. The effects of both relaxation time and parameter $\beta$ are found to be weak on this field. The values of thermodynamical temperature get slightly decreased in the context of fractional order strain.

The variations of conductive temperature distribution with spatial coordinate $x$ are described in Figure 4. As expected, the conductive temperature has the same starting value zero for all the three cases which is in good agreement

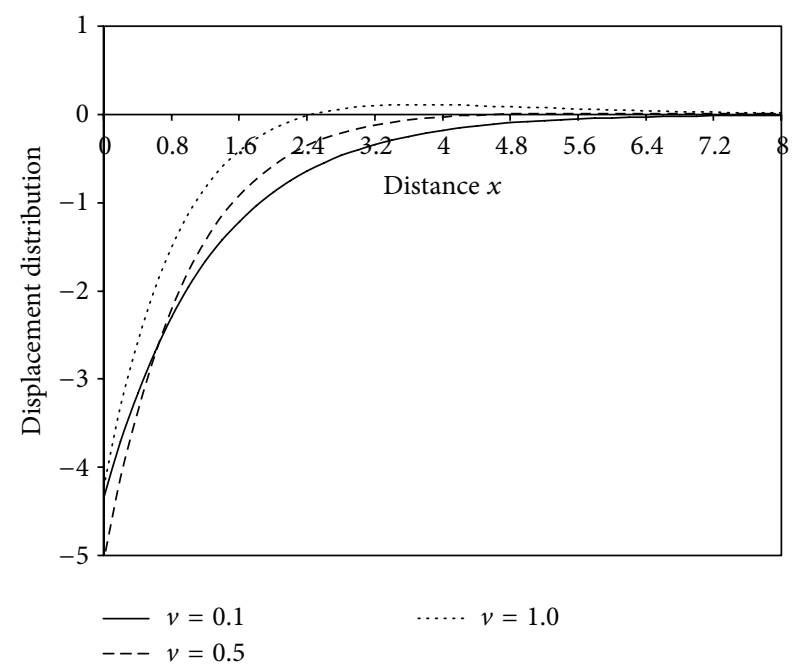

FIGURE 5: Dependence of displacement distribution on velocity $v$ of heat source.

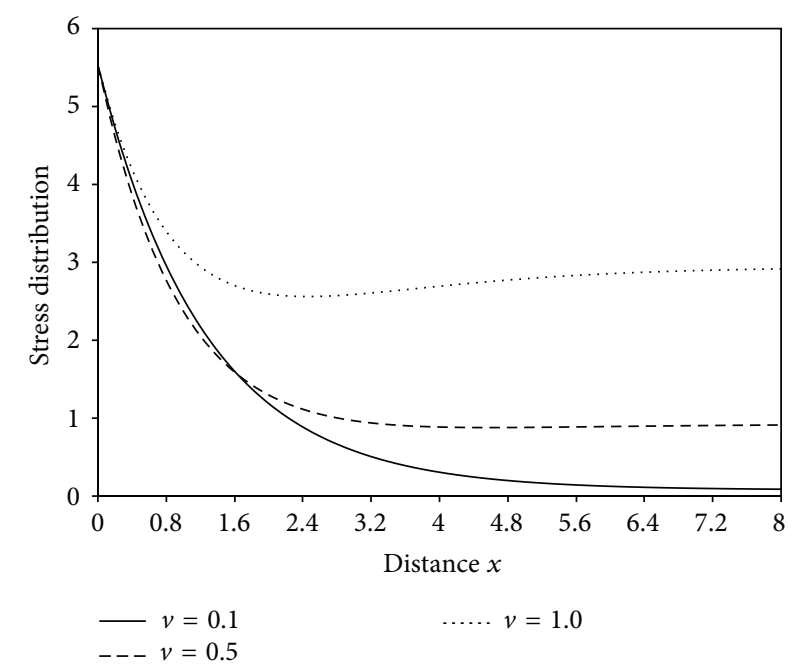

Figure 6: Dependence of stress distribution on velocity $v$ of heat source.

with the boundary conditions. No significant difference in the value of thermodynamical temperature is noticed for $\tau=0$ (without fractional order strain) and $\tau=0.01$ (with fractional order strain).

Group II. The dependence of displacement field on the velocity of internal heat source is shown in Figure 5. The effect of velocity $v$ is very much noticeable near the point of application of the source and it dies out with increase in the spatial coordinate. Figure 6 demonstrates a comparison of the stress distribution for three different values of velocity of heat source. The pertinent effects of velocity $v$ are noticed in the range $1.6 \leq z \leq 8$. In this range, an increase in the velocity gives a significant increase in the values of stress.

Variations in the thermodynamical temperature $\theta$ for different values of velocity $v$ are depicted in Figure 7 . The profile indicates that the velocity of heat source has a 


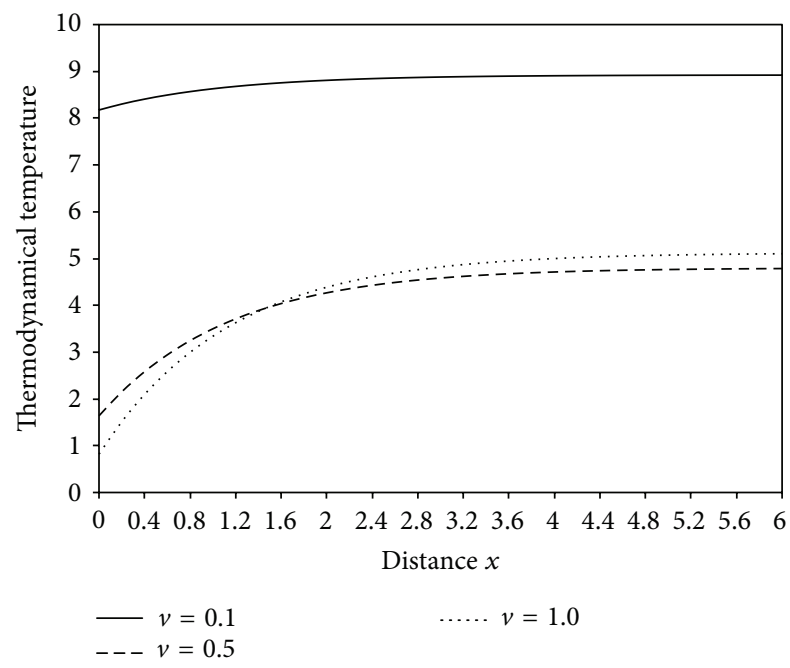

FIGURE 7: Dependence of thermodynamical temperature on velocity $v$ of heat source.

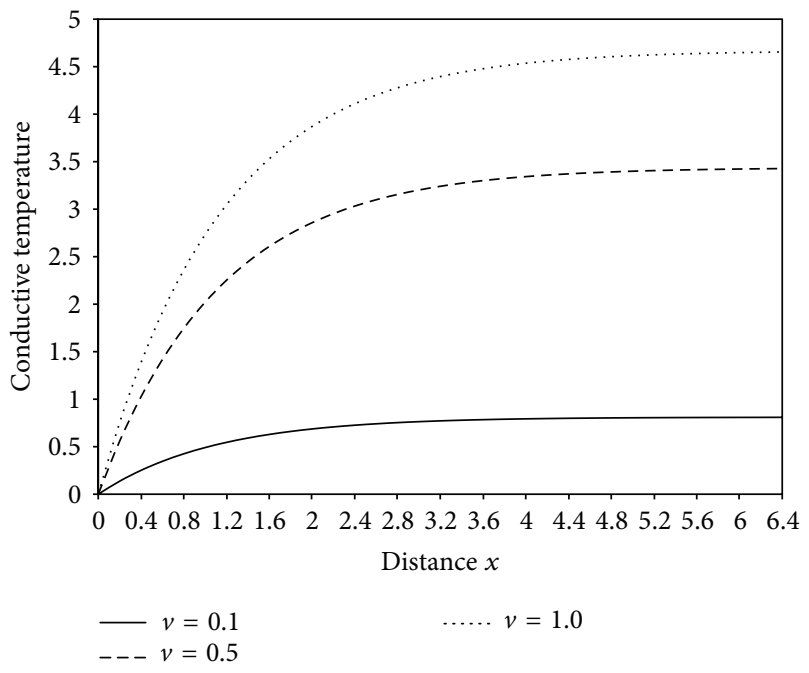

Figure 8: Dependence of conductive temperature on velocity $v$ of heat source.

pronounced effect on the thermodynamical temperature $\theta$. The conductive temperature distribution is represented graphically in Figure 8 at different values of velocity $v$. The qualitative behavior of conductive temperature is the same for all the three values of $v$. An increase in the velocity $v$ leads to a significant increase in the values of conductive temperature.

Group III. Figure 9 displays the influence of viscosity and twotemperature parameter on the spatial variations of displacement distribution $u$. For all the three cases, the displacement distribution starts with its maximum negative values and then approaches zero gradually. The numerical values of displacement in TV2T case are found to be smaller than that in TV1T medium and this difference is highly noticeable near the point of application of the source. Viscosity has a little impact on this distribution. The dynamic effects of viscosity and two-temperature parameter on the stress distribution

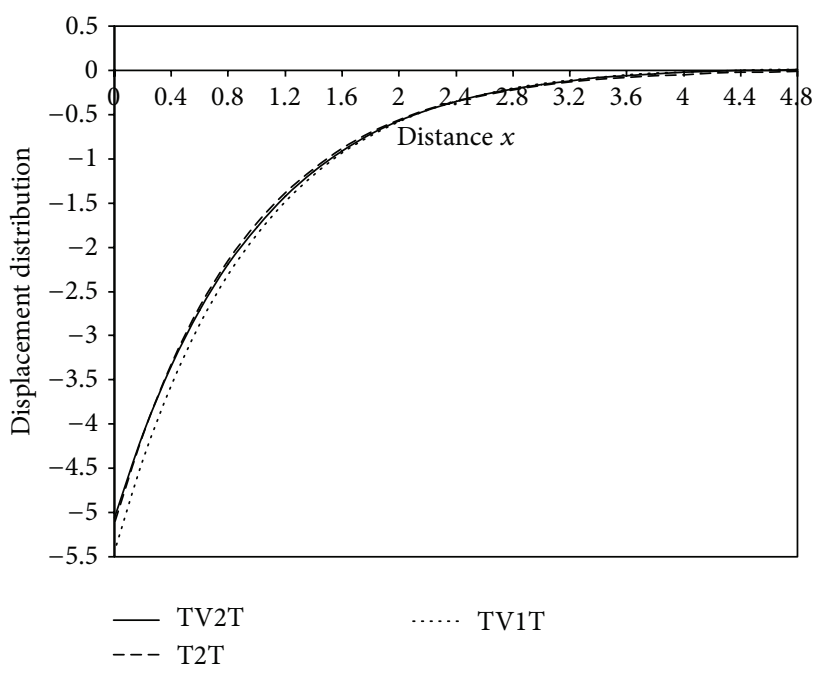

FIGURE 9: Effects of viscosity and two-temperature parameter on displacement distribution.

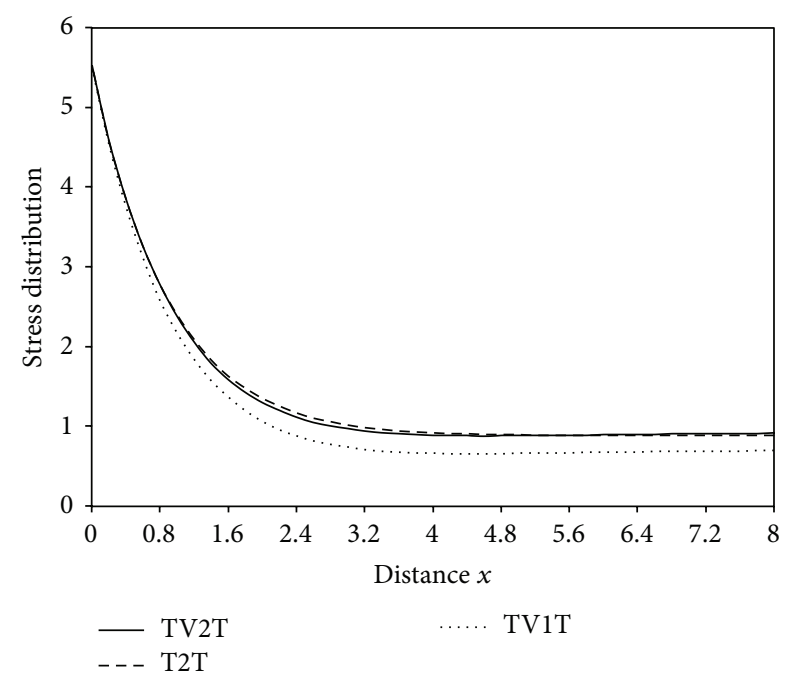

FIGURE 10: Effects of viscosity and two-temperature parameter on stress distribution.

are examined in Figure 10. The presence of two-temperature parameter has caused an increment in the numerical values of this field. The effect of two-temperature parameter is found to be more profound than the effect of viscosity.

The distribution of thermodynamical temperature $\theta$ for three different cases is shown in Figure 11. Values of thermodynamical temperature are more in the context of two-temperature thermoelasticity as compared to onetemperature thermoelasticity pointing towards the significant increasing effect of two-temperature parameter. For the viscous solid, values of this distribution are found to be smaller than that of a nonviscous solid. This distribution experiences a similar pattern of variations for all the three cases irrespective of their magnitude.

The dependence of conductive temperature $\varphi$ on twotemperature parameter and viscosity is exhibited in Figure 12. 


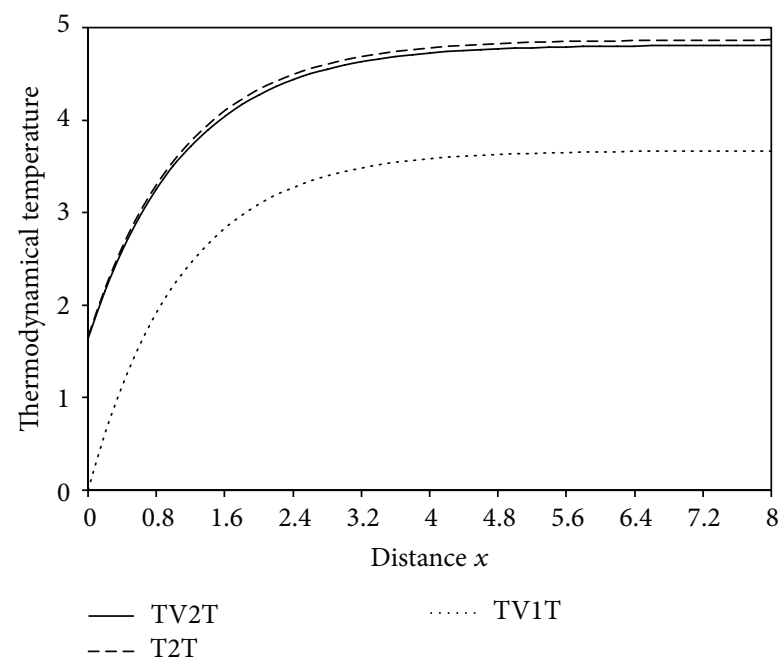

FIGURE 11: Effects of viscosity and two-temperature parameter on thermodynamical temperature.

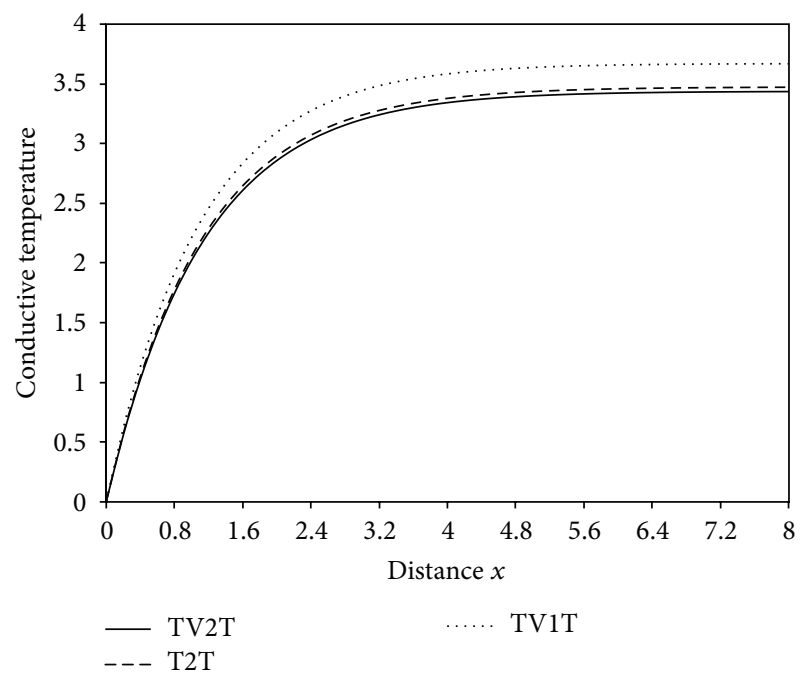

FIGURE 12: Effects of viscosity and two-temperature parameter on conductive temperature.

This field starts with value zero, increases sharply in the range $0 \leq z \leq 2.4$, and then increases slowly in the rest of the range for all the three cases. The figure suggests that the two-temperature parameter acts to decrease the values of conductive temperature. It can be noted from the figure that the values of conductive temperature differ slightly for TV2T and T2T media which signifies the little impact of viscosity on this field.

Group IV. Figures 13-16 display the variations of considered physical variables in the presence and absence of heat source. With reference to Figures 13 and 14, it is found that the presence of heat source has caused both increasing and decreasing effects on the displacement and stress distributions. On the other hand, both thermodynamical and conductive temperature distributions are significantly increased due to presence of heat source, as can be seen from the plots

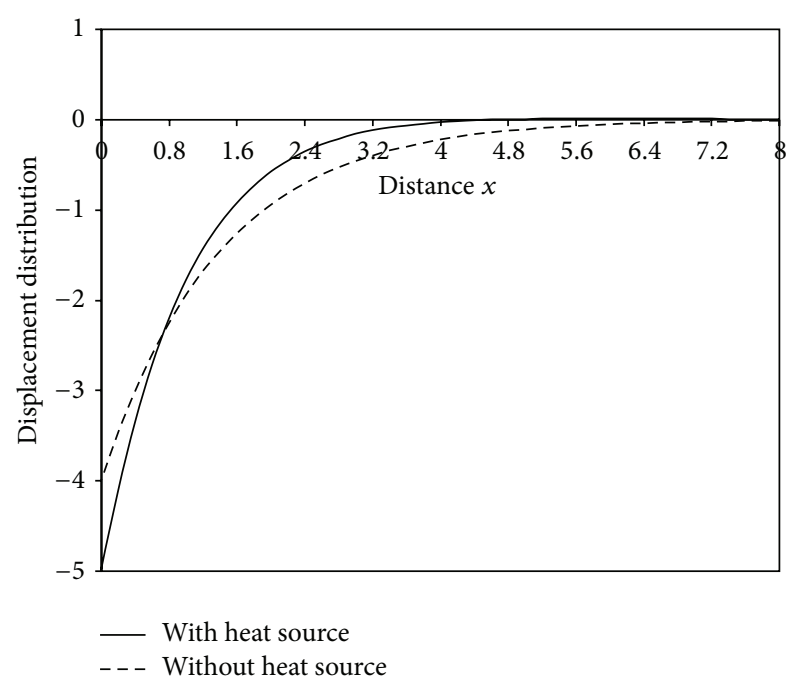

FIGURE 13: Distribution of displacement with distance $x$.

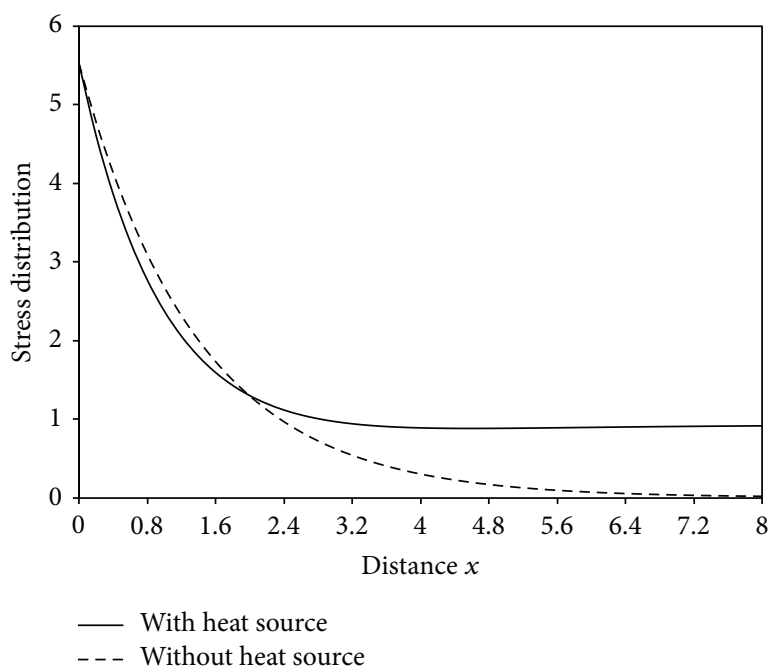

FIgURE 14: Distribution of stress with distance $x$.

in Figures 15 and 16. From Figures 13-16, we observe that all the considered fields diminish to zero with increasing values of $x$ in the medium without internal heat source.

\section{Conclusions}

The effects of mechanical relaxation time, fractional strain parameter, viscosity, heat source, and two-temperature parameter in a homogeneous and isotropic elastic solid due to a mechanical load have been investigated by applying the recently developed theory of generalized thermoelasticity with fractional order strain. The method of Laplace transform is used to write the basic equations in the form of vector-matrix differential equation which is then solved by state-space approach. The numerical inversion of Laplace transform is carried out using Fourier series expansion technique. The present analysis leads to the following concluding remarks: 


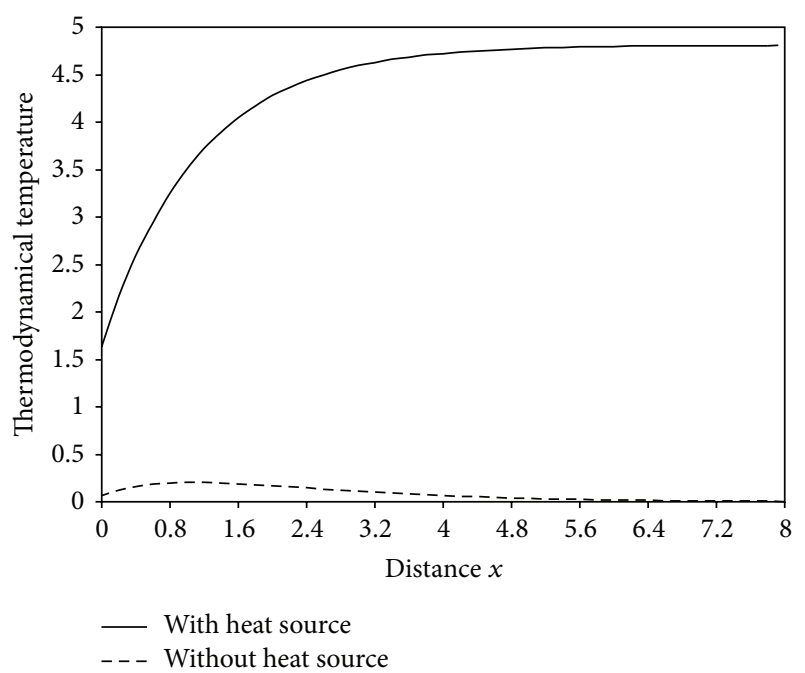

Figure 15: Distribution of thermodynamical temperature with distance $x$

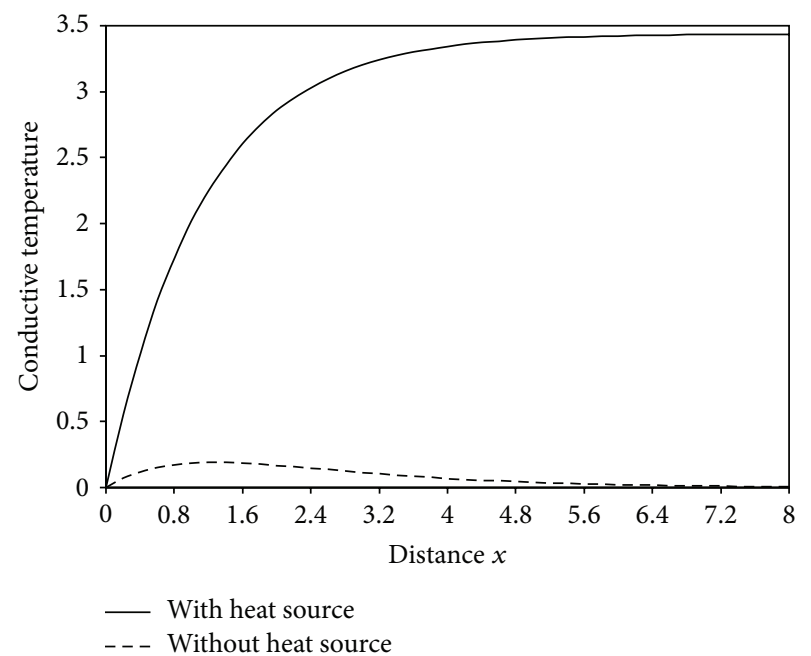

FIGURE 16: Distribution of conductive temperature with distance $x$.

(1) The nonzero value of mechanical relaxation time $\tau$ significantly influences the variations of displacement and stress. However it has a little effect on the variations of thermodynamical temperature and conductive temperature.

(2) The displacement and stress distributions are found to be more sensitive towards the fractional strain parameter $\beta$ than the temperature distributions.

(3) The velocity of heat source is playing a vital role in increasing or decreasing the speed of wave propagation through the elastic medium.

(4) Presence of viscosity has caused a little impact on all the studied fields.

(5) Two-temperature parameter has affected all the considered physical variables. It acts to increase the magnitude of stress and thermodynamical temperature distributions while it has decreased the magnitude of displacement and conductive temperature distributions.

(6) The presence of internal heat source has an essential role in changing the values of the distributions. The displacement and stress fields have received both increasing and decreasing effects due to heat source while the magnitude of thermodynamical and conductive temperatures gets enlarged in the presence of heat source.

(7) It is apparent from the figures that the presence of heat source prevents all the considered fields except displacement from becoming zero with distance $x$. In the absence of heat source, all the physical fields have nonzero values in a bounded region of space and, outside this region, values vanish identically manifesting the phenomenon of finite speed of propagation.

The present work describes the behavior of displacement, stress, thermodynamical temperature, and conductive temperature in a thermoviscoelastic medium with twotemperature and internal heat source due to mechanical shock under the purview of generalized thermoelasticity with fractional order strain. This work may prove helpful in biomechanics and biomedical problems and in understanding the viscoelastic properties of human soft tissue and may lead to improved diagnostic applications.

\section{Conflict of Interests}

The authors declare that there is no conflict of interests regarding the publication of this paper.

\section{References}

[1] H. W. Lord and Y. Shulman, "A generalized dynamical theory of thermoelasticity," Journal of the Mechanics and Physics of Solids, vol. 15, no. 5, pp. 299-309, 1967.

[2] A. E. Green and K. A. Lindsay, "Thermoelasticity," Journal of Elasticity, vol. 2, no. 1, pp. 1-7, 1972.

[3] A. E. Green and P. M. Naghdi, "A re-examination of the basic postulate of thermo-mechanics," Proceedings of the Royal Society London A, vol. 432, pp. 171-194, 1991.

[4] A. E. Green and P. M. Naghdi, "On undamped heat waves in an elastic solid," Journal of Thermal Stresses, vol. 15, no. 2, pp. 253-264, 1992.

[5] A. E. Green and P. M. Naghdi, "Thermoelasticity without energy dissipation," Journal of Elasticity, vol. 31, no. 3, pp. 189-208, 1993.

[6] P. J. Chen and M. E. Gurtin, "On a theory of heat conduction involving two temperatures," Zeitschrift für angewandte Mathematik und Physik, vol. 19, no. 4, pp. 614-627, 1968.

[7] P. J. Chen and W. O. Williams, "A note on non-simple heat conduction," Zeitschrift für angewandte Mathematik und Physik, vol. 19, no. 6, pp. 969-970, 1968.

[8] P. J. Chen, M. E. Gurtin, and W. O. Williams, "On the thermodynamics of non-simple elastic materials with two temperatures," Zeitschrift für Angewandte Mathematik und Physik, vol. 20, no. 1, pp. 107-112, 1969.

[9] B. A. Boley and I. S. Tolins, "Transient coupled thermoelastic boundary value problems in the half-space," Journal of Applied Mechanics, vol. 29, no. 4, p. 637, 1962. 
[10] W. E. Warren and P. J. Chen, "Wave propagation in the two temperature theory of thermoelasticity," Acta Mechanica, vol. 16, no. 1-2, pp. 21-33, 1973.

[11] H. M. Youssef, "Theory of two-temperature-generalized thermoelasticity," IMA Journal of Applied Mathematics, vol. 71, no. 3, pp. 383-390, 2006.

[12] H. M. Youssef and E. A. Al-Lehaibi, "State-space approach of two-temperature generalized thermoelasticity of onedimensional problem," International Journal of Solids and Structures, vol. 44, no. 5, pp. 1550-1562, 2007.

[13] H. M. Youssef, "Theory of two-temperature thermoelasticity without energy dissipation," Journal of Thermal Stresses, vol. 34, no. 2, pp. 138-146, 2011.

[14] S. Deswal and K. K. Kalkal, "Fractional order heat conduction law in micropolar thermo-viscoelasticity with two temperatures," International Journal of Heat and Mass Transfer, vol. 66, pp. 451-460, 2013.

[15] A. M. Zenkour and A. E. Abouelregal, "State-space approach for an infinite medium with a spherical cavity based upon twotemperature generalized thermoelasticity theory and fractional heat conduction," Zeitschrift fur Angewandte Mathematik und Physik, vol. 65, no. 1, pp. 149-164, 2014.

[16] M. I. A. Othman and M. I. M. Hilal, "Rotation and gravitational field effect on two-temperature thermoelastic material with voids and temperature dependent properties type III," Journal of Mechanical Science and Technology, vol. 29, no. 9, pp. 37393746, 2015.

[17] M. B. Bera, N. C. Das, and A. Lahiri, "Eigenvalue approach to two-temperature generalized thermoelastic interactions in an annular disk," Journal of Thermal Stresses, vol. 38, no. 11, pp. 1310-1324, 2015.

[18] B. Ross, "The development of fractional calculus 1695-1900," Historia Mathematica, vol. 4, no. 1, pp. 75-89, 1977.

[19] K. S. Miller and B. Ross, An Introduction to the Fractional Calculus and Fractional Differential Equations, John Wiley \& Sons, New York, NY, USA, 1993.

[20] I. Podlubny, Fractional Differential Equations, Academic Press, New York, NY, USA, 1999.

[21] Y. Z. Povstenko, "Fractional heat conduction equation and associated thermal stress," Journal of Thermal Stresses, vol. 28, no. 1, pp. 83-102, 2005.

[22] M. Caputo, "Linear model of dissipation whose Q is almost frequency independent-II," Geophysical Journal International, vol. 13, no. 5, pp. 529-539, 1967.

[23] Y. Z. Povstenko, "Fractional radial heat conduction in an infinite medium with a cylindrical cavity and associated thermal stresses," Mechanics Research Communications, vol. 37, no. 4, pp. 436-440, 2010.

[24] H. H. Sherief, A. M. A. El-Sayed, and A. M. Abd El-Latief, "Fractional order theory of thermoelasticity," International Journal of Solids and Structures, vol. 47, no. 2, pp. 269-275, 2010.

[25] H. M. Youssef, "Theory of fractional order generalized thermoelasticity," Journal of Heat Transfer, vol. 132, no. 6, pp. 1-7, 2010.

[26] S. Deswal and K. K. Kalkal, "Two temperature magnetothermoelasticity with initial stress: state space formulation," Journal of Thermodynamics, vol. 2013, Article ID 754798, 12 pages, 2013.

[27] S. Deswal and K. K. Kalkal, "Plane waves in a fractional order micropolar magneto-thermoelastic half-space," Wave Motion, vol. 51, no. 1, pp. 100-113, 2014.
[28] I. A. Abbas, "Eigen value approach to fractional order generalized magneto-thermoelastic medium subjected to moving heat source," Journal of Magnetism and Magnetic Materials, vol. 377, pp. 452-459, 2015.

[29] S. Santra, N. C. Das, R. Kumar, and A. Lahiri, "Threedimensional fractional order generalized thermoelastic problem under the effect of rotation in a half space," Journal of Thermal Stresses, vol. 38, no. 3, pp. 309-324, 2015.

[30] Y. Z. Wang, D. Liu, Q. Wang, and J. Z. Zhou, "Fractional order theory of thermoelasticity for elastic medium with variable material properties," Journal of Thermal Stresses, vol. 38, no. 6, pp. 665-676, 2015.

[31] H. M. Youssef, "Theory of generalized thermoelasticity with fractional order strain," Journal of Vibration and Control, 2015.

[32] V. Danilovskaya, "Thermal stresses in an elastic semi-space due to a sudden heating of its boundary," Prikladnaya Matematika $i$ Mekhanika, vol. 14, pp. 316-318, 1950.

[33] G. Eason and I. N. Sneddon, "X.-the dynamic stresses produced in elastic bodies by uneven heating," Proceedings of the Royal Society of Edinburgh Section A: Mathematical and Physical Sciences, vol. 65, no. 2, pp. 143-176, 1959.

[34] W. Nowacki, "Some dynamic problems of thermoelasticity," Archiwum Mechaniki Stosowanej, vol. 9, pp. 325-334, 1959.

[35] H. H. Sherief and M. N. Anwar, "Problem in generalized thermoelasticity," Journal of Thermal Stresses, vol. 9, no. 2, pp. 165-181, 1986.

[36] A. M. Freudenthal, "Effect of rheological behavior on thermal stresses," Journal of Applied Physics, vol. 25, no. 9, pp. 1110-1117, 1954.

[37] A. A. Ilioushin and B. E. Pobedria, Fundamentals of the Mathematical Theory of Thermal Viscoelasticity, Nauka, Moscow, Russia, 1970.

[38] S. Mukhopadhyay, "Effects of thermal relaxations on thermoviscoelastic interactions in an unbounded body with a spherical cavity subjected to a periodic loading on the boundary," Journal of Thermal Stresses, vol. 23, no. 7, pp. 675-684, 2000.

[39] A. Baksi and R. K. Bera, "Relaxation effects on plane wave propagation in a rotating magneto-thermo-viscoelastic medium," Journal of Thermal Stresses, vol. 29, no. 8, pp. 753-769, 2006.

[40] A. Kar and M. Kanoria, "Generalized thermo-visco-elastic problem of a spherical shell with three-phase-lag effect," Applied Mathematical Modelling, vol. 33, no. 8, pp. 3287-3298, 2009.

[41] S. Deswal and K. Kalkal, "A two-dimensional generalized electro-magneto-thermoviscoelastic problem for a half-space with diffusion," International Journal of Thermal Sciences, vol. 50, no. 5, pp. 749-759, 2011.

[42] S. Deswal and R. Yadav, "Thermodynamic behaviour of microstretch viscoelastic solids with internal heat source," Canadian Journal of Physics, vol. 92, no. 5, pp. 425-434, 2014.

[43] G. Honig and U. Hirdes, "A method for the numerical inversion of Laplace transforms," Journal of Computational and Applied Mathematics, vol. 10, no. 1, pp. 113-132, 1984. 


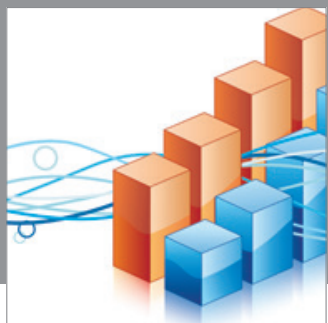

Advances in

Operations Research

mansans

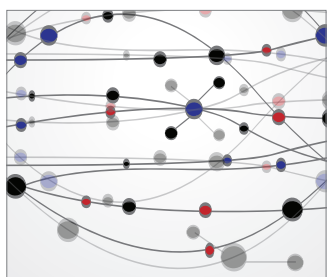

The Scientific World Journal
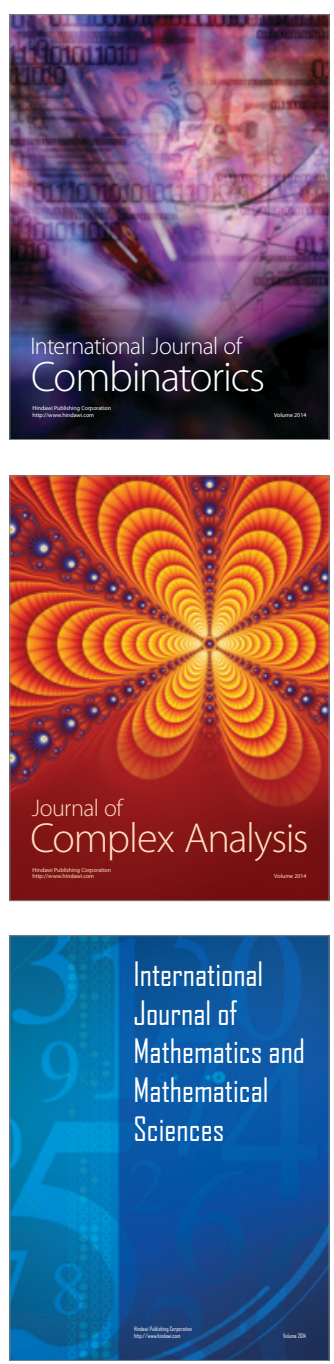
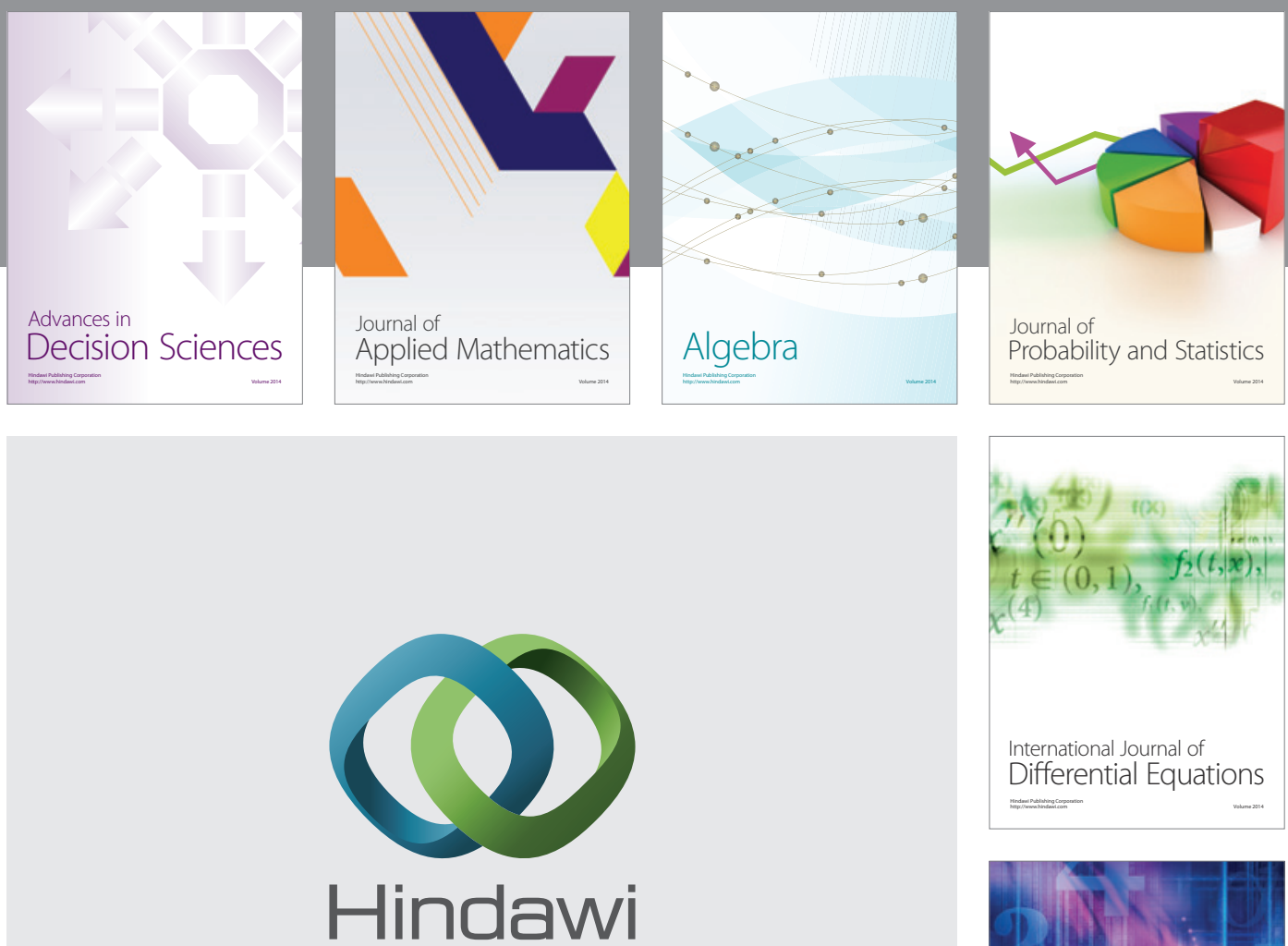

Submit your manuscripts at http://www.hindawi.com
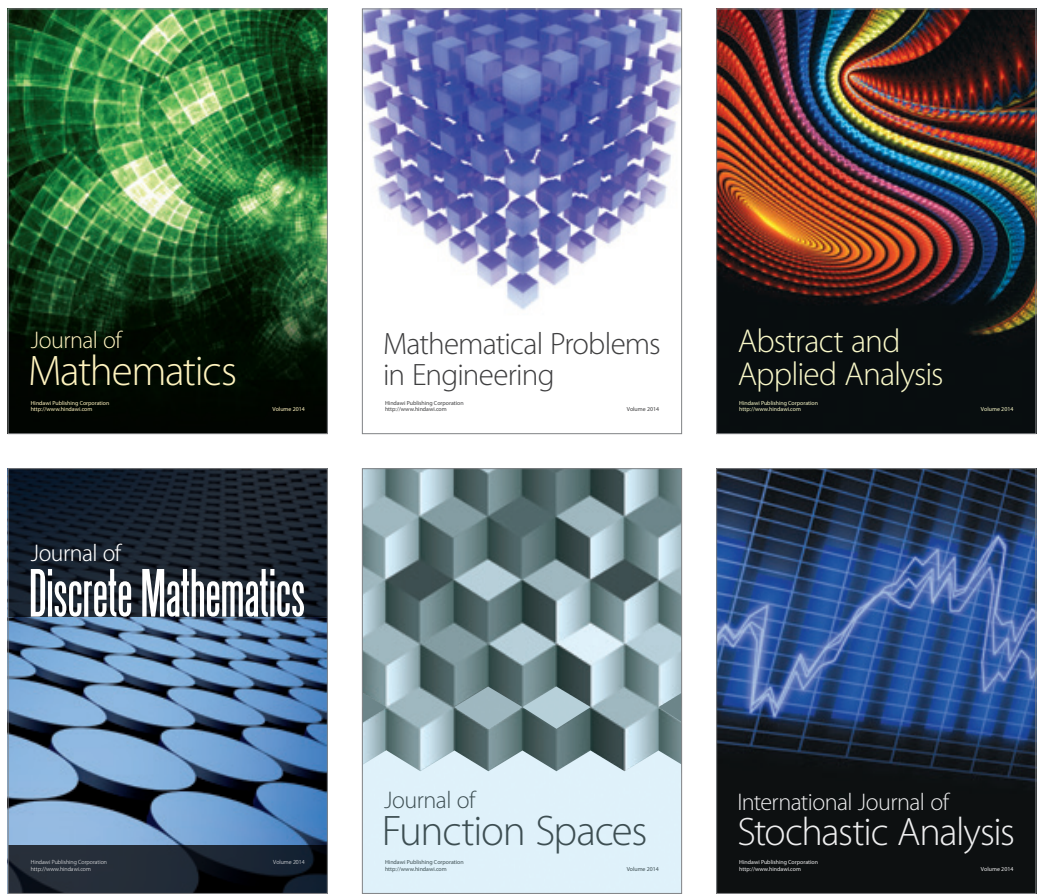

Journal of

Function Spaces

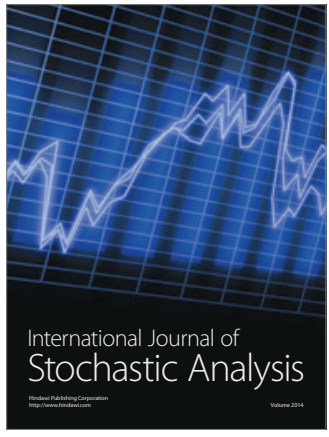

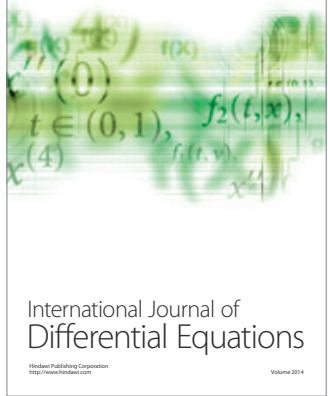
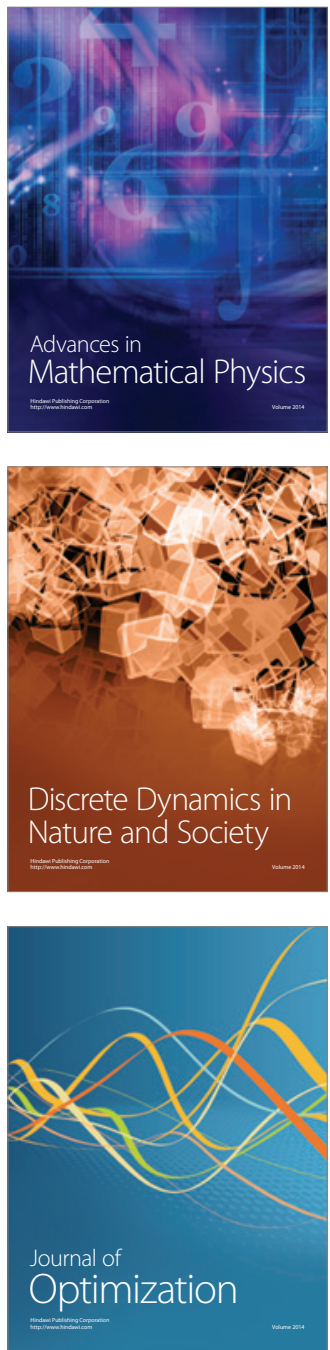